\title{
Diagnosis of Cysticercosis in post auricular swelling on FNAC- A case report
}

\author{
Prashant V. Kumavat ${ }^{1, *}$, Anjali Mahajan'2, Chetan S Chaudhari ${ }^{3}$, Ganesh Kshirsagar ${ }^{4}$, \\ Poorva Rane ${ }^{5}$ \\ ${ }^{1,3,4}$ Assistant Professor, ${ }^{2}$ Associate Professor, ${ }^{5}$ Resident, Dept. of Pathology, LTMMC \& LTMGH, Sion, Mumbai, \\ Maharashtra, India
}

*Corresponding Author:

Email: drkumavat_83@rediffmail.com

\begin{abstract}
Introduction: Cysticercosis, a parasitic tissue infection is caused by larva of Taenia solium (Cysticercus cellulosae). Fine needle aspiration cytology (FNAC) provides direct, specific, simplest and early diagnosis of cysticercosis and remains one of the ideal diagnostic procedure wherever the lesion can be approached easily.

Case Report: We present a case of 21 year old female presented with swelling on left postauricular area for one month, which was $2 \times 1 \mathrm{~cm}$, firm, tender and mobile. USG was suggestive of infective aetiology. FNAC was done using 24-gauge needle. Whitish blood mixed aspirate was seen. Smears were wet fixed with $95 \%$ ethyl alcohol and stained with Papanicolaou stain. Cytology smears showed fragments of bluish fibrillary material with interspersed small nuclei, which were seen against background of mixed inflammatory infiltrate of neutrophils, histiocytes, lymphocytes and eosinophils. Essential for diagnosis of cysticercosis are identification of parasitic fragments which may comprise bluish, fibrillary structures, sometimes with honeycombing, calcospherules, teguments, scolex with hooklets and hyaline membrane surrounding it.

Conclusion: Fine needle aspiration cytology is a low cost, outpatient procedure which helps in early diagnosis of cysticercosis. A careful search for parasitic fragments is to be made in presence of polymorphous inflammatory infiltrate composed predominantly of neutrophils, histiocytes and eosinophils.
\end{abstract}

Keywords: Cysticercosis, Cytology, Inflammation, Fibrillary material.

Received: $24^{\text {th }}$ May, 2017

\section{Introduction}

Cysticercosis is a potentially dangerous systemic zoonotic disease. It is an infection of larval form of pork tapeworm of Taenia solium. ${ }^{[1]}$ The word "Cysticercus" is derived from two Greek words, "Kystis" meaning cyst and "Kertos" meaning tail due to its appearance. ${ }^{2}$ These diseases are endemic in America, Asia and Africa. It is more common in northern parts of India. Frequents sites of involvement are brain, subcutaneous tissue, skeletal muscle, and eye. ${ }^{3}$ Humans may be accidental or incidental host of Taenia solium by drinking contaminated water or consuming undercooked pork that harbour the larva. ${ }^{4}$ Consumption of raw vegetables such as radish, cabbage and carrots which are infected with eggs may also lead to disease. ${ }^{4}$

Fine needle aspiration cytology (FNAC) is a well-recognized preoperative low cost direct, specific and simplest diagnostic procedure for the evaluation of inflammatory nodules caused by parasites. ${ }^{4} \mathrm{We}$ present a case of cysticercosis in a post auricular swelling on cytology.

\section{Case Report}

We present a case of 21 years old female presented with swelling on left post auricular area for one month which measures $2 \times 1 \mathrm{~cm}$ subcutaneous, firm, tender and mobile. USG showed $7.3 \times 6.2 \times 5.2 \mathrm{~mm}$ well defined hypoechoic lesion showing an eccentric hyperechoic lesion suggestive of infective aetiology. FNAC was done using 24-gauge needle. Material aspirated from lesion was white in colour and presence of blood tinge was noted. Smears were wet fixed with 95\% ethyl alcohol (hair spray) and stained with Papanicoloau stain.

Cytology smears showed fragments of larva thrown into many rounded folds and bluish fibrillary material with interspersed small nuclei (1-2 $\mu$ ) (Fig. 1 A, B). Fragments of parasite were seen against background of mixed inflammatory infiltrate comprising of neutrophils, eosinophils, lymphocytes and histiocytes (Fig. 1C). A final diagnosis offered was subcutaneous cysticercosis on cytology which was confirmed on histopathology. On histopathology, it shows cystic cavity contain larva of cysticercosis. It has cephalic and caudal end. Caudal end reveals duct like invagination which is lined by double layered, homogenous eosinophilic membrane. Cysticercosis has outer cuticle layer which is smooth and hyalinized. Beneath the tegument is a row of tegumental cells. The inner fibrillary layer or parenchyma is loose and reticular, containing mesenchymal cells and calcerous corpuscles. 
Surrounding inflammation marked with double arrow head. (Fig. 2)

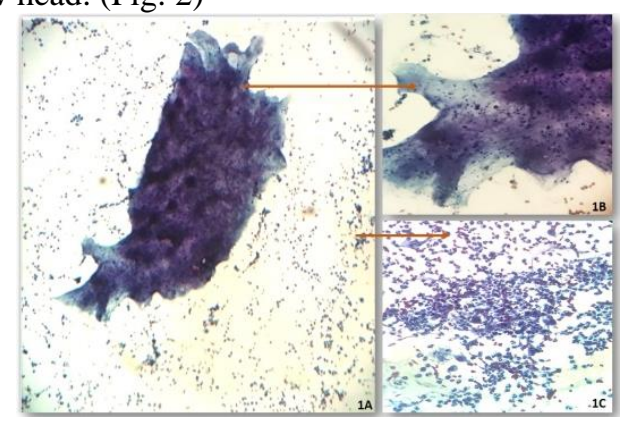

Fig. 1:

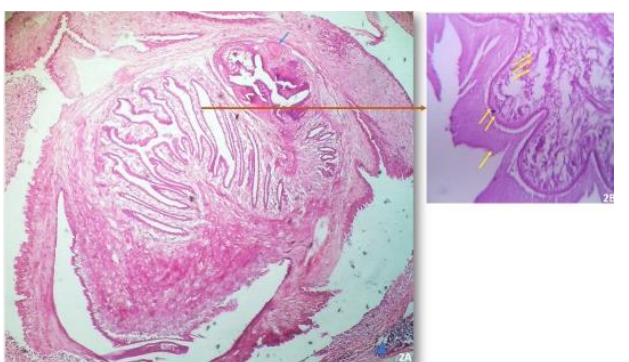

Fig. 2:

\section{Discussion}

Worldwide cysticercosis is a not uncommon infestation. There is $7 \%$ to $26 \%$ prevalence range of porcine cysticercosis in India. In humans, there is no data or study revealing prevalence of cysticercosis in India. ${ }^{5}$ The pig is an intermediate host and human is a definite host. Cysticercosis is caused by the larval form of Taenia solium that is, Cysticercus cellulosae. The adult worm present in the small intestine of humans where it is attached with scolex and sheds eggs or gravid segments through faeces on the ground.

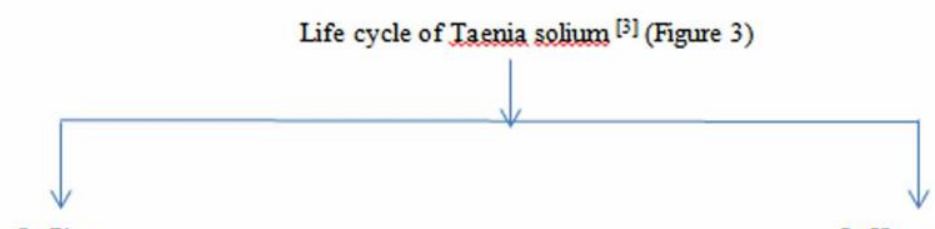

In Pigs

While grazing pigs swallow the eggs or gravid segment in faeces

Reaches alimentary canal of pig and the oncospheres are released by rupture of eggs

oncospherespenetrate the gut wall via the hooklets.

Enter systemic circulation by Breaching portal vessels or mesenteric lymph nodes<smiles>C1CCC1</smiles>

Reaches musculartissue and Ulimately settles down

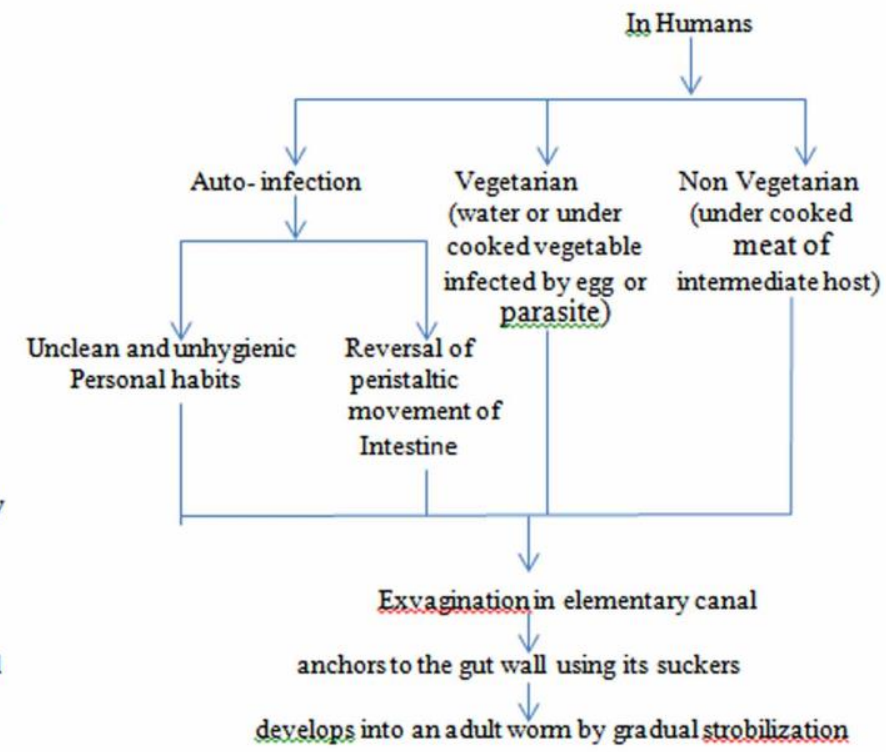




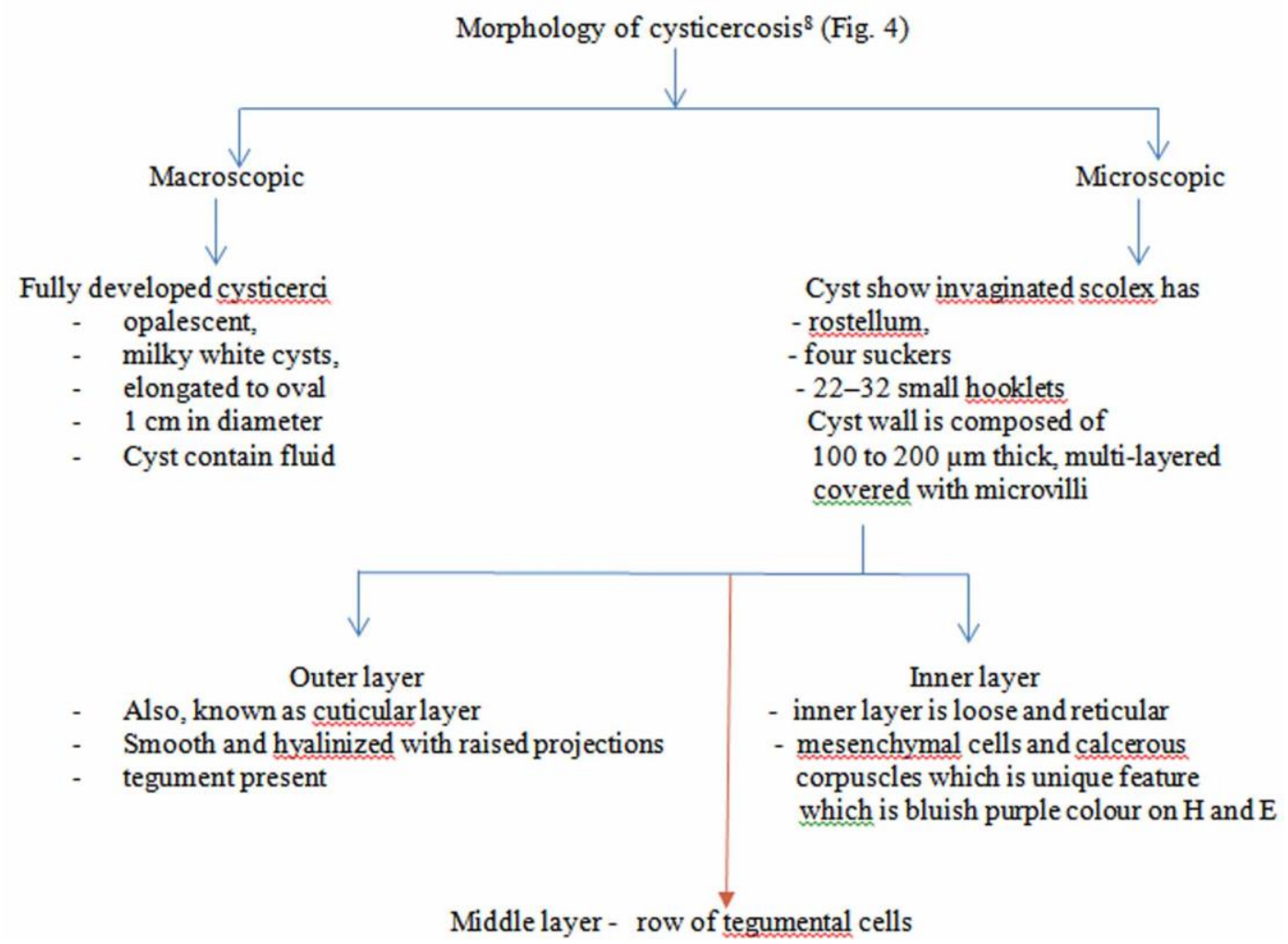

Cysticercosis can be diagnosed by different diagnostic methods such as serology, radiology, and pathological examination. ${ }^{11}$ For CNS involvement MRI and CT scan are more sensitive in diagnosing cysticercosis but they are very costly and provide only supportive diagnosis. Serological tests has significance only when they are positive. Negative results cannot rule out cysticercosis and in addition false positivity results can be seen due to cross reactivity with other helminth infection in past. That is why FNAC is common pre-operative tool for the diagnosis of cysticercosis. ${ }^{11}$

Cyticerci nodules of subcutaneous location can be misinterpreted as lymphadenitis or benign mesenchymal tumours on clinical grounds. ${ }^{9}$ Parasitic lesion should be suspected in subcutaneous nodule, once cytologist starts seeing neutrophils, eosinophil, histiocytes and giant cells. Once fragments of parasite, calcareous corpuscles and hooklets is seen diagnosis is gets confirmed. Scolex is difficult to find on FNAC. ${ }^{10}$ In our case body wall structure such as fibrillar bladder wall with numerous dots (nuclei) (Fig. 1) were noted which is sufficient for diagnosis, no scolex or hooklets were seen in our case. Shobhitha D suggest that a definitive diagnosis of cysticercosis can be given only when parasite structure is seen. ${ }^{5}$ On FNAC only $12 \%$ cases showed hooklets but the majority of cases i.e., $62.2 \%$ reviewed in literature showed the presence of structure of parasite. If none such features are present and inflammatory infiltrate may also be variable, subsequent

histopathological examination helps in pinpointing diagnosis which was also done in this case.

The literature studied at aspiration reveals clear fluid in $30.2 \%$ cases, and purulent fluid was in $10 \%$ of the cases. The undisturbed bladder with minimal surrounding inflammation usually contains clear fluid. When the bladder wall is overtaken by inflammation, then the inflammatory exudates may contaminate the clear fluid resulting in a purulent aspirate. ${ }^{5}$ In our case aspirate was whitish with presence of blood tinge, whitish due to inflammation and haemorrhagic may be due to FNAC procedure.

\section{Conclusion}

Fine-needle aspiration cytology is cheaper and reliable investigation in cysticercosis for early detection and management. Cysticercosis is common in India so it should be kept in mind in all inflammatory and cystic lesions.

\section{Reference}

1. Bhattacharjee H, Ramman T, Argarwal L, Agarwal L, Nain M, Thomas S. Isolated cysticercosis of the breast masquerading as a breast tumour: report of a case and review of literature. Annals of Tropical 
Medicine \& Parasitology. 2011 Sep 1;105 (6):455-

61.

2. Koteeswaran G, Mangala G, Kotasthane D, Tirou A. Cysticercosis of tongue: Cytohistologic approach to diagnosis. Journal of oral and maxillofacial pathology: JOMFP. 2013 Sep;17(3):480.

3. Chatterjee D. Parasitology - Protozoology and Helminthology. 13th ed. New Delhi: CBS Publishers and Distributors Pvt. Limited;2014.

4. Gill M, Dua S, Gill P, Gupta V, Gupta S, Sen R. Cytomorphological spectrum of subcutaneous and intramuscular cysticercosis: A study of 22 cases. Journal of cytology. 2010 Oct 1;27(4):123.

5. Shobhitha D, Shariff S. Diagnosis of Cysticercosis by Fine Needle Aspiration Cytology: Report of Four Cases with a Review of Literature in the Indian Subcontinent. Journal of Medical Sciences and Health. 2016 Sep;2(3):13-18.

6. Vuong P. Fine needle aspiration cytology of subcutaneous cysticercosis of the breast. Case report and pathogenic discussion. Acta cytologica. 1988 Dec;33(5):659-62.

7. Kamal M, Grover S. Cytomorphology of subcutaneous cysticercosis. A report of 10 cases. Acta cytologica. 1994 Dec;39(4):809-12.

8. Ash L, Orihel T. Larval cestode parasite in humans. Atlas of human parasitology. 3rd ed Chicago: IL: American Society of Clinical Pathologists 1990.P.236-7.

9. Kung I, Lee D, Yu H. Soft tissue cysticercosis: diagnosis by fine-needle aspiration. American journal of clinical pathology. 1989 Dec 1;92(6):8345.

10. Verma K, Kapila K. Fine needle aspiration diagnosis of cysticercosis in soft tissue swellings. Acta cytologica. 1988 Dec;33(5):663-6.

11. Handa U, Garg S, Mohan H. Fine needle aspiration in the diagnosis of subcutaneous cysticercosis. Diagnostic cytopathology. 2008 Mar 1;36(3):183-7. 